\title{
Growing fatty mass in the back: diagnosis of a multiple symmetric lipomatosis (Madelung's disease) in association with chronic alcoholism
}

\author{
Andreas Gutzeit • Christoph A. Binkert • \\ Sina Schmidt • Abdul R. Jandali • Jochen Mutschler • \\ Klaus Hergan • Sebastian Kos
}

Received: 28 February 2011 /Revised: 6 September 2011 /Accepted: 7 September 2011 /Published online: 11 November 2011

(C) ISS 2011

\section{Part 1: question}

Growing fatty mass in the back.

A 50-year-old man with chronic alcoholism was referred with clinical signs of a slowly growing fatty

The diagnosis can be found at doi:10.1007/s00256-011-1280-6.

A. Gutzeit $(\bowtie) \cdot$ C. A. Binkert

Department of Radiology, Cantonal Hospital Winterthur,

Brauerstrasse 15,

8401 Winterthur, Switzerland

e-mail: Andreas.Gutzeit@ksw.ch

A. Gutzeit $\cdot$ K. Hergan

Department of Radiology,

Paracelsus Medical University Salzburg,

Salzburg, Austria

S. Schmidt $\cdot$ A. R. Jandali

Departement of Surgery, Cantonal Hospital Winterthur,

Brauerstrasse 15,

8401 Winterthur, Switzerland

J. Mutschler

Department of General and Social Psychiatry, Psychiatric

University Hospital Zurich,

Militärstrasse 8,

CH-8021 Zürich, Switzerland

S. Kos

Institute of Radiology and Nuclear Medicine,

University hospital Basel,

Petersgraben 4 ,

4031 Basel, Switzerland mass in the back developing over the previous 3 years (Fig. 1). Additional fatty tumors were noted in the neck (Figs. 2a, b), and in the upper parts of the arms and the thighs (Fig. 3a, b). Cytology results showed no signs of malignancy (Fig. 4).

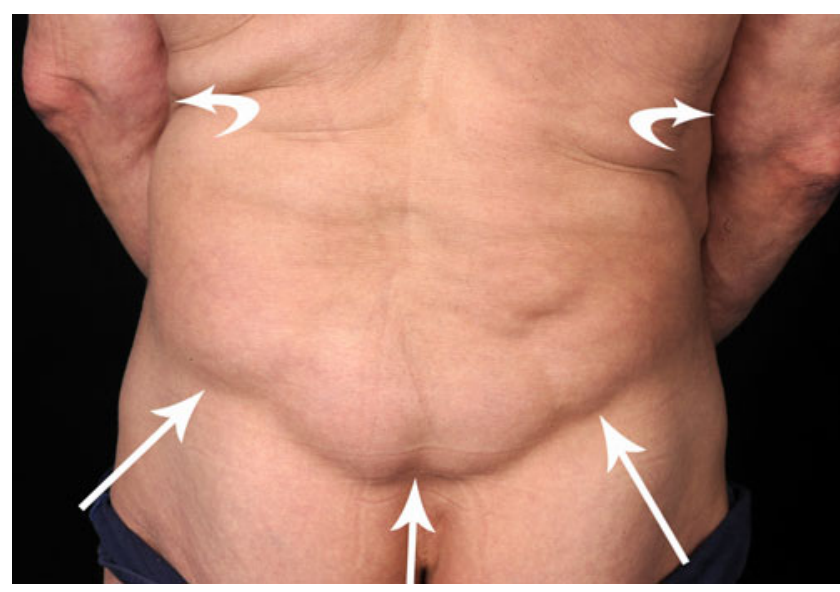

Fig. 1 A $20 \times 20 \mathrm{~cm}$ mass is visible in the lower back (arrows). The mass had no secondary signs of inflammation, was soft on palpation and subjectively painless and non-tender. Additional tumors can be clearly seen around the elbow (curved arrows) 

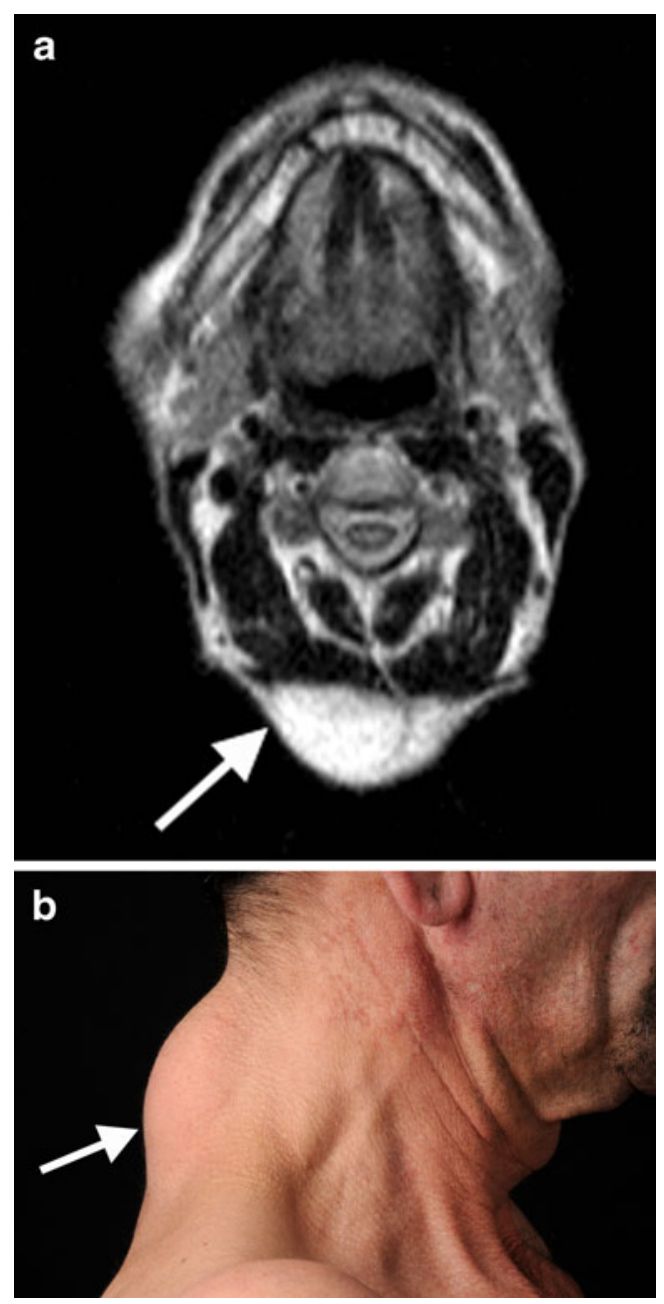

Fig. 2 a Axial T2-weighted sequence from a whole-body MRI shows subcutaneous fatty infiltration (arrow) in the right dorsal neck. b Photograph corresponding to a, showing the mass (arrow)

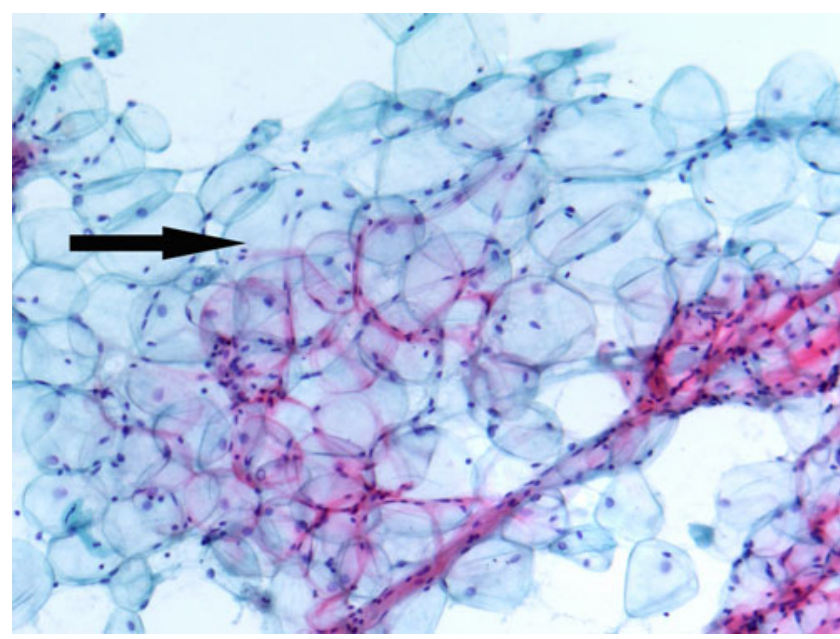

Fig. 4 Aspiration cytology from the fatty mass in the dorsal neck showed normal fatty tissue without any signs of malignancy with abundant normal lipoid cells (arrow). (Original magnification $\times 100$, Papanicoloau stain)

Acknowledgements We want to thank the Department of Pathology of Cantonal Hospital Winterthur, especially Dr. Ivo Tosony, for helping us in the preparation of the histological image.
Fig. 3 In this $\mathrm{T} 1$ sequence, there is diffuse subcutaneous fatty infiltration in the thigh, especially on the right side (arrow)
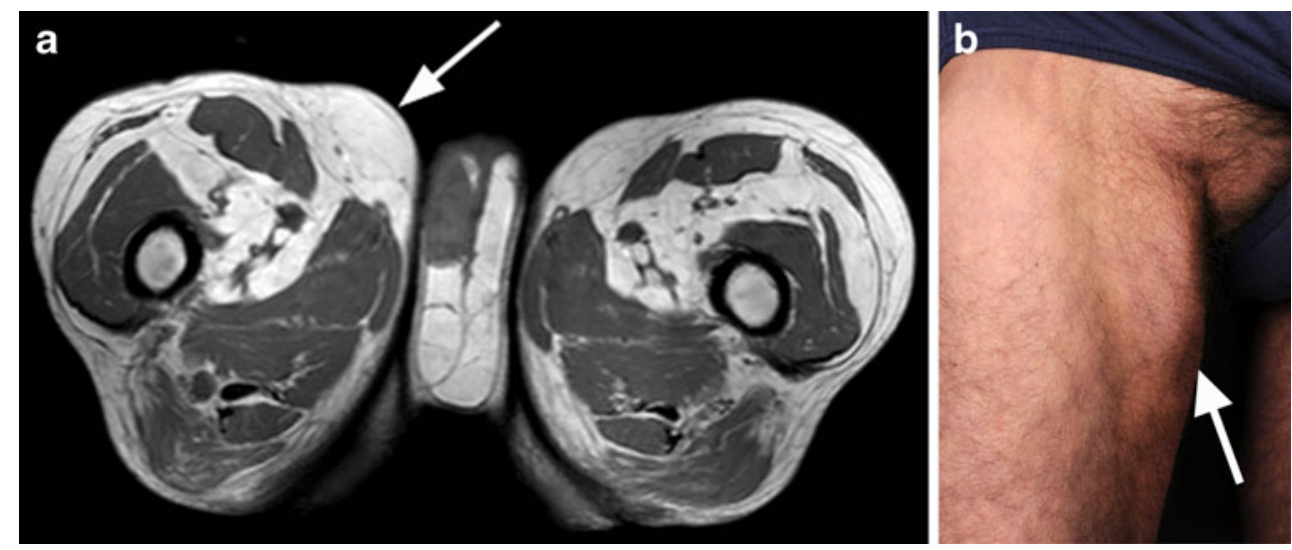